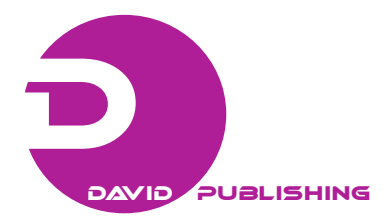

\title{
MCNPX Modeling of BN-600 Full MOX Fast Reactor Core
}

\author{
Moustafa Aziz and Mohga Hassan \\ Nuclear and Radiological Regulatory Authority, Nasr City, Cairo 11762, Egypt
}

Received: June 05, 2013 / Accepted: July 07, 2013 / Published: August 25, 2013.

\begin{abstract}
The BN-600 full mixed oxide fueled core is modeled using MCNPX code. A three dimensional model typical of the core is prepared which includes 6 radial zones, low enrichment zone, medium enrichment zone and high enrichment zone, two zones of steel shielding assemblies, and a radial blanket in addition to shim and scram rods. The twelve axial zones are also modeled. The model calculates core parameters such as reactor multiplication factor, power distribution through reactor core, and control rod worth. An estimation of isotope transmutation through various reactor zones and analysis of internal breeding zone were presented. The results are compared with previously published results which indicated good agreement. Typical results of the present model for reactor multiplication factor are 1.00832 compared with transport model (1.00664) and diffusion model (1.00131). The high enrichment zone dominates power production. The results also estimate the isotope variation due to burnup in the MOX core.
\end{abstract}

Key words: BN-600, full MOX, MCNPX, power production, isotope transmutation.

\section{Introduction}

A benchmark analysis of a BN-600 fully mixed oxide core design with sodium plenum above the core has been performed as an extension to the study of BN-600 hybrid uranium oxide [1]. Compared with the hybrid core model, several design modifications have been made in the full mixed oxide (MOX) fueled core model. A sodium plenum followed by a boron shield is located above the core to reduce the sodium void effect. An internal breeding zone (IBZ) of $5.1 \mathrm{~cm}$ thickness is inserted in the mid plane to achieve the reduction of sodium void effect [1]. To compensate for the reduction of core volume resulted from these design changes, an extra row of FSA is added in the medium enrichment zone (MEZ) region. The same geometry description for fuel subassemblies (FSAs) and control rods have been retained in a triangular lattice.

In this work the BN-600 full MOX core is modeled using Monte Carlo n-particle extended (MCNPX) code [2]. Core multiplication factor and control rod

Corresponding author: Mohga Hassan, Dr., research fields: nuclear design and radiation shielding. E-mail: mohgaig@yahoo.com. worth are estimated. Power generation in various zones is evaluated. The concentration variation with operating time for important isotopes is estimated and the IBZ is analyzed.

\section{Core and Model Description}

MCNPX code, based on Monte Carlo method, is used to design a three dimensional model typical of the reactor core. All core zones radially and axially are represented in the model. The reactor fuel consists of Mixed Oxide $\left(\mathrm{PuO}_{2}+\mathrm{UO}_{2}\right)$ and the core consists of six radial zone: low enrichment zone (LEZ), medium enrichment zone (MEZ), high enrichment zone (HEZ), followed by two zones of steel shielding assemblies (SSA), and finally radial reflector.

In addition there is also 19 shim and control rods (SHR), and 6 scram rods (SCR).

The core axially consists of different axial zones from the bottom of the reactor reflector, axial blanket, core internal breeding zone, core, plugs, sodium plenum, cones upper boron shield, cones and reflector, respectively.

All fuel isotopes are modeled at a uniform temperature of $1,500 \mathrm{~K}$ and all structural and coolant isotopes are at a uniform temperature of $600 \mathrm{~K}$. The 
material compositions of isotopes specified for each zone are given in Ref. [3]. The active core height is $87.4 \mathrm{~cm}$ including IBZ which exists at the center of LEZ only with active thickness $5.1 \mathrm{~cm}$ with composition different from other fuel zone area. The total core height is $204 \mathrm{~cm}$, and the average core radius through horizontal plan is $158.432 \mathrm{~cm}$.

Fig. 1a illustrates typical horizontal layout of the model. Fig. 1b illustrates typical vertical layout of the model.

\section{Results and Discussion}

\subsection{Calculation of Reactor Multiplication Factor}

Table 1 illustrates the reactor multiplication factor at start up of the reactor (zero burnup). The given values for transport and diffusion models are the average values for different organizations and Labs contributed to this project [4]. The difference between present model and transport and diffusion models is $\Delta k=0.00168$ and 0.00701 , respectively. The results indicate that transport models are in close agreement with present MCNPX models than diffusion methods. Fig. 2 illustrates reactor multiplication factor $\left(\mathrm{K}_{\mathrm{eff}}\right)$ versus operation time, the results indicate that $\mathrm{K}_{\mathrm{eff}}$ drops quickly at start due to fission product build up and then decreases linearly

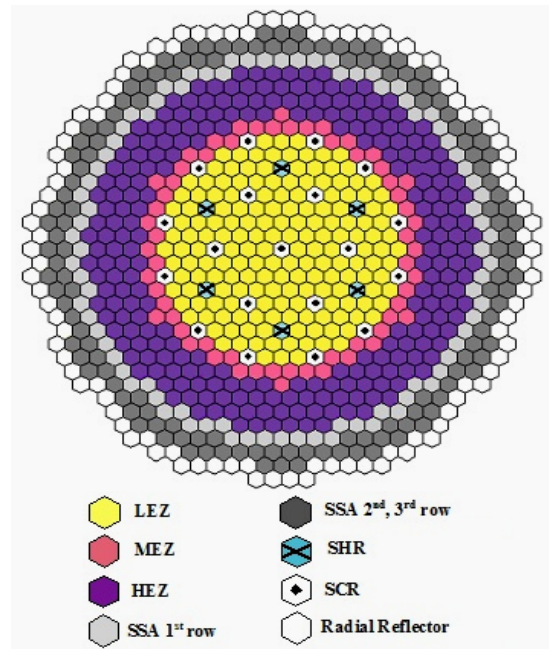

(a) with time.

\subsection{Power Generation Through Different Zones}

Table 2 illustrates the power generation at different zones inside the core, HEZ dominate power generation by producing $45 \%$ of the total power, LEZ produces $37.71 \%$ and MEZ produces $13.89 \%$. IBZ produces $1.22 \%$, while little power is produced at axial blanket 1 and 2 .

The results show good agreement with the Ref. [5]. The maximum difference occurs at LEZ 5.1\%. The power is calculated at zero burn up.

\subsection{Control Rod Worth Analysis}

The reactor core contains 19 SHR rods which are used to fine and coarse reactivity and power control and 6 SCR rods which are used for reactor scram, the reactor multiplication factor is calculated at different rods positions.

Table 3 summarizes the results of this study. From these results we can conclude the following : The core excess reactivity is 0.033396 . Total SHR reactivity worth is 0.056896 . Total control rods SCR reactivity worth is 0.038846 , these results indicate that the ratio of total SHR worth to core excess reactivity equals 1.703677 and SCR rods shutdown the reactor core because SCR worth exceeds core excess reactivity at

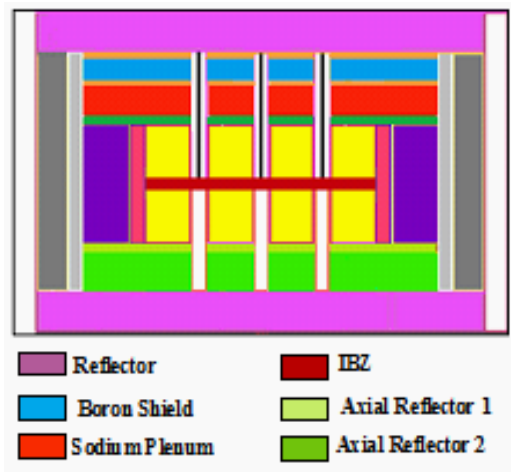

(b)

Fig. 1 Typical Layout of BN-600 Full MOX Computer Model. (a) radial layout and (b) axial layout. 
Table 1 Reactor multiplication factor at zero burnup.

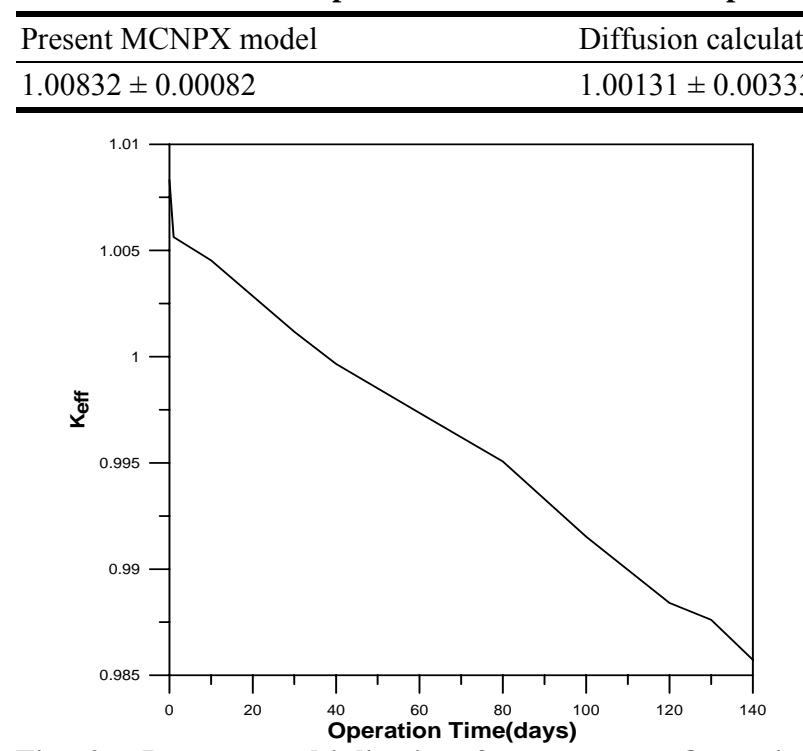

Fig. 2 Reactor multiplication factor versus Operation Time (days).

cold state without xenon effect.

\subsection{Isotope Transmutation}

The change in the concentration of fissile isotopes was also investigate in this study.

Fig. 3 illustrates ${ }^{235} \mathrm{U}$ concentrations versus operation time. From the figure it can be seen that the ${ }^{235} \mathrm{U}$ concentration decreased in all reactor zones, this decrease is about $15.6 \%$ in the LEZ zone and $14 \%$ in both MEZ and HEZ zones, while it is about is $12 \%$ in the axial blanket.
Transport calculations [4]

$1.00664 \pm 0.00071$

The change in the concentration of ${ }^{239} \mathrm{Pu}$ is illustrated in Fig. 4. The results indicate that $\mathrm{Pu}-239$ concentration decreases in LEZ, MEZ and HEZ while increases in axial blanket. The decrease is about $4 \% 5 \%$ $4.6 \%$ in LEZ, MEZ, HEZ respectively, while the increase in axial Blanket is about $77.6 \%$. This indicates that ${ }^{239} \mathrm{Pu}$ builds up significantly in axial blanket.

Fig. 5 illustrates ${ }^{241} \mathrm{Pu}$ concentrations versus operation time. The results indicate that ${ }^{241} \mathrm{Pu}$ builds up in all zones of the reactor core. The percentage increase in LEZ MEZ and HEZ are 38\%, 31.5\%, $23.6 \%$, respectively and $195 \%$ for the axial blanket.

\subsection{Internal Breeding Zone (IBZ)}

Changes in the content of fissile isotopes in IBZ are illustrated in Figs. 6, 7, and 8. It can be seen that ${ }^{235} \mathrm{U}$ concentration decreases with operation time due to its burn up. The concentration decreases from $2.255 \times$ $10^{-5}$ for fresh fuel to $1.835 \times 10^{-5}$ after 140 days which means that $18.62 \%$ of ${ }^{235} \mathrm{U}$ are consumed inside the IBZ. On the other hand ${ }^{239} \mathrm{Pu}$ concentration increases from $3.747 \times 10^{-4}$ to $4.832 \times 10^{-4}$ which results in buildup of about $30 \%{ }^{239} \mathrm{Pu}$ in IBZ.

Table 2 Power generation through different zones inside the core.

\begin{tabular}{lcll}
\hline Zone & Present model Power MW & Percent Contribution & Reference [5] \\
\hline LEZ & 554.48 & 0.3771 & 526.18 \\
MEZ & 204.18 & 0.1389 & 204.39 \\
HEZ & 661.5 & 0.45 & 690.07 \\
IBZ & 17.96 & 0.01222 & 17.78 \\
Axial Blanket 1 & 12.055 & 0.0082 & 11.54 \\
Axial Blanket 2 & 19.757 & 0.0134 & 20.03 \\
\hline Total & 1470 & 1.0 & \\
\hline
\end{tabular}

Table $3 \mathrm{~K}_{\text {eff }}$ at Different Control Rod Position.

\begin{tabular}{ll}
\hline SCR and SHR positions & $\mathrm{K}_{\text {eff }}$ \\
\hline SHR and SCR fully out & $1.03455 \pm 0.00047$ \\
SHR fully IN and SCR fully out & $0.97704 \pm 0.00037$ \\
SHR in Mid Core and SCR fully out & $1.00775 \pm 0.00037$ \\
SHR fully OUT and SCR fully in & $0.99458 \pm 0.00036$ \\
\hline
\end{tabular}




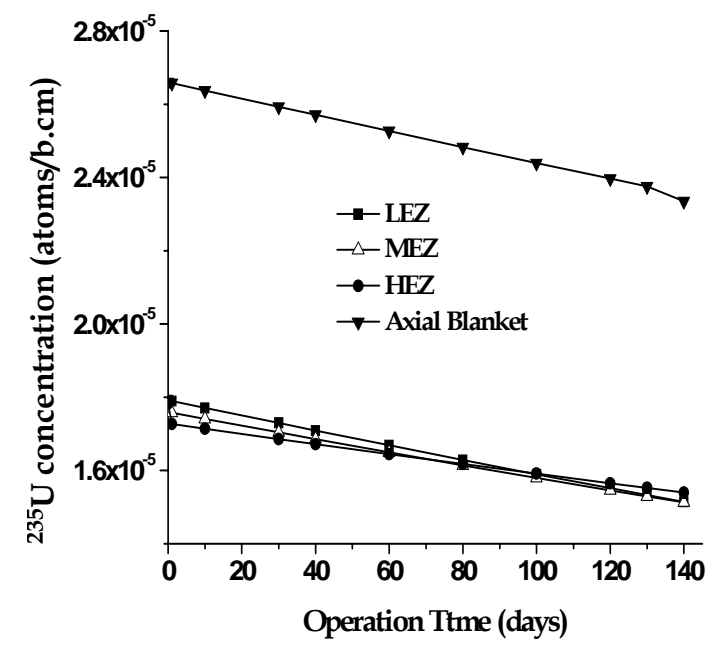

Fig. $3{ }^{235} \mathrm{U}$ concentrations at different zones.

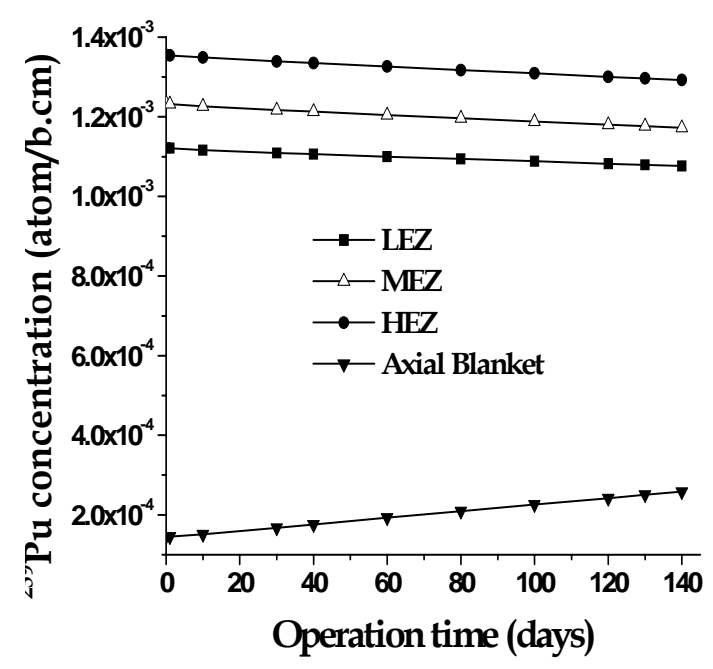

Fig. $4{ }^{239} \mathrm{Pu}$ concentrations at different zones.

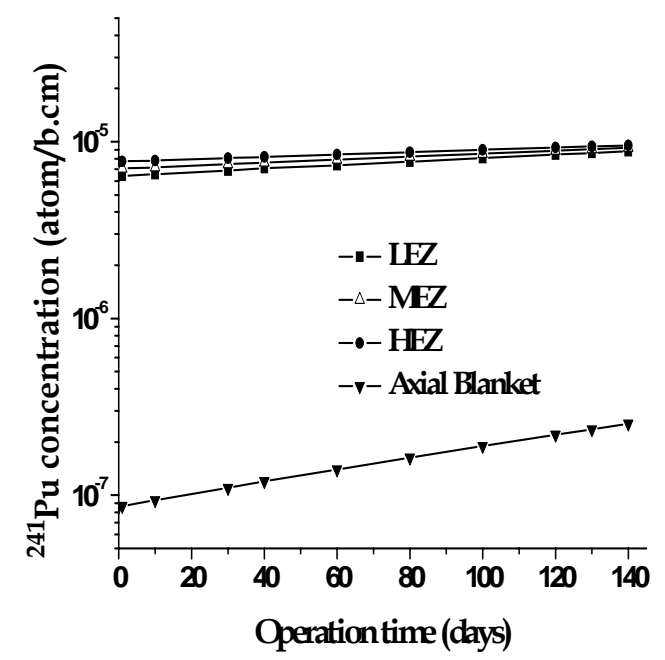

Fig. $5{ }^{241} \mathrm{Pu}$ concentrations at different zones 3.5 internal breeding zone (IBZ).

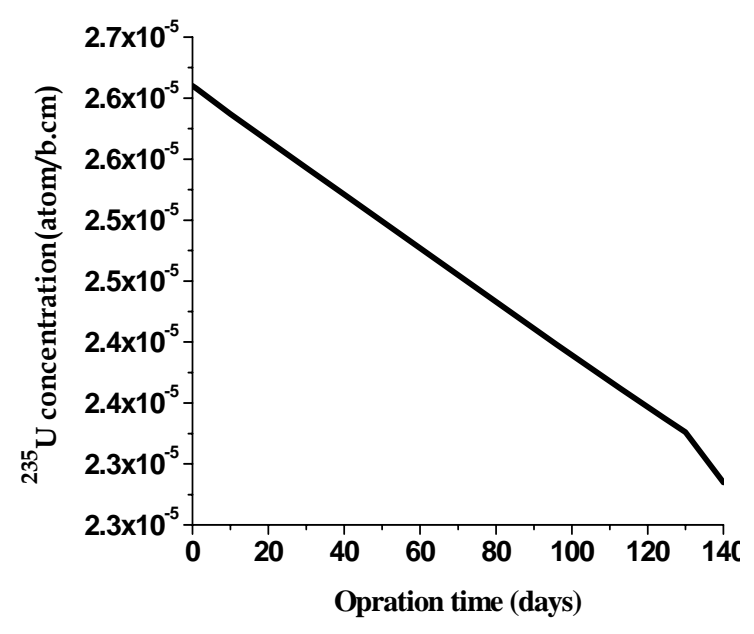

Fig. $6{ }^{235} \mathrm{U}$ concentration versus operation time in IBZ.

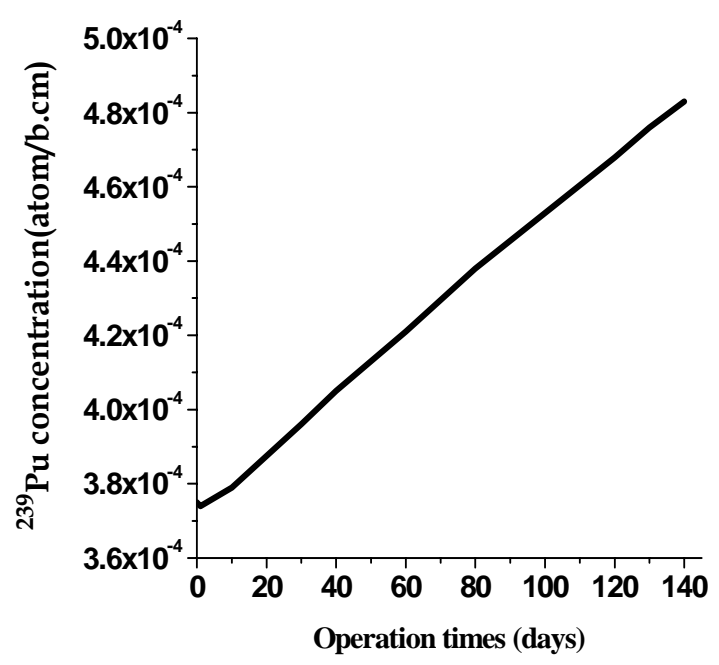

Fig. $7{ }^{239} \mathrm{Pu}$ concentration versus operation time in IBZ.

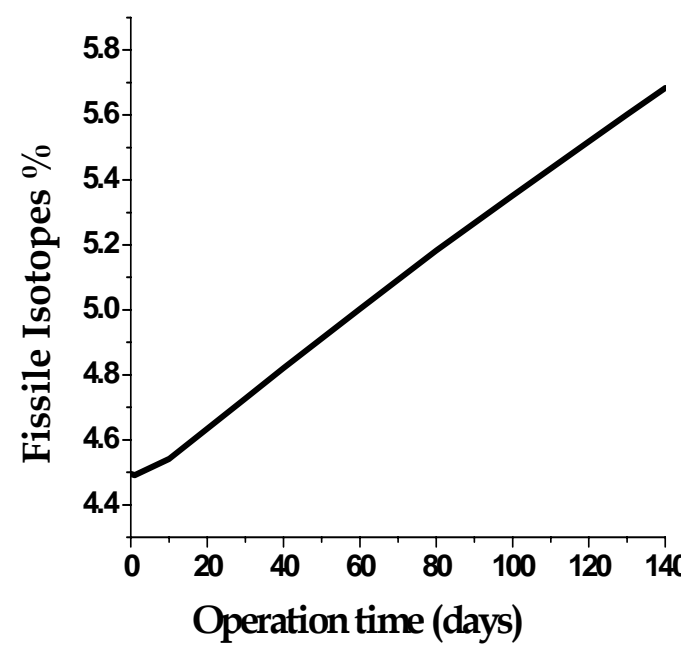

Fig. 8 Ratio of fissile isotopes versus operation time at IBZ. 
Fig. 8 illustrates the ratio of fissile isotopes ${ }^{235} \mathrm{U}+$ ${ }^{239} \mathrm{Pu}+{ }^{241} \mathrm{Pu}$ ) over the total concentration (fissile + fertile) versus operation time the results indicate that the ratio increases with time from $4.495 \%$ to $5.683 \%$ at 140 day of irradiations.

\section{Conclusions}

The BN-600 full MOX core is modeled using MCNPX code. The results are compared with previously published papers which indicated good agreement.

HEZ dominate power generation by producing $45 \%$ of the total power, LEZ produces $37.71 \%$, MEZ produces $13.89 \%$, and IBZ $1.22 \%$.

Total SHR reactivity worth is 0.056896 , while total SCR reactivity worth is 0.038846 .

${ }^{235} \mathrm{U}$ concentrations decreases, with operation in all reactor zones, the maximum percentage of consumption is $18.62 \%$ in IBZ.

${ }^{239} \mathrm{Pu}$ builds up in the core and its increase ranges from $4 \%$ in LEZ to more than $77 \%$ in axial blanket.

The ratio of fissile isotopes $\left({ }^{235} \mathrm{U}+{ }^{239} \mathrm{Pu}+{ }^{241} \mathrm{Pu}\right)$ over the total concentration (fissile + fertile) versus operation time (day) increases with time from $4.495 \%$ to $5.683 \%$ at 140 day of irradiations.

\section{References}

[1] Y.I. Kim, R. Hill, K. Grimm, G. Rimpault, T. Newton, Z. H. Li, et al., BN-600 full MOX core benchmark analysis, in proceeding: PHYSOR 2004: The Physics of Fuel Cycles and Advanced Nuclear Systems: Global Developments, Chicago, 2004, 104-123.

[2] J.S. Hendricks, G.W. Mckinney, M.L. Fensin, M.R. James, R.C. Johns, J.W. Durkee, MCNPX version 2.6E, Los Alamos National Lab, USA, 2007.

[3] V.Y. Stogov, V.A. Cherny, A.V. Danylitchev, I.Y. Krivitski, Specification of Phase 4 Benchmark, (Hex-Z fully Mox fuelled Core Model of BN-600), Updated Codes and Methods to Reduce Calculational Uncertainities of Liquid Metal Fast Reactor Reactivity Effects, IAEA-RC-803.5, TWG-FR/120, IAEA Vienna, 2004, pp. 331-334.

[4] Updated Codes and Methods to reduce the Calculational Uncertainties of the LMFR Reactivity Effects, in: Second research coordination Meeting, IAEA-RC-803.5, TWG-FR/120, IAEA Vienna, 2004,108.

[5] M. Ishikawa, JNC Results of BN 600 Benchmark Calculation (phase 4) Specification of Phase 4 Benchmark, (Hex-Z fully Mox fuelled Core Model of BN-600), Updated Codes and Methods to Reduce the Calculational Uncertainities of Liquid Metal Fast Reactor Reactivity Effects IAEA-RC-803.5, TWG-FR/120, IAEA Vienna, 2004, pp. 175-179. 